\title{
Addition of routine blood biomarkers to TIMI risk score improves predictive performance of 1-year mortality in patients with ST-segment elevation myocardial infarction
}

Pyung Chun Oh ${ }^{1 \dagger}$, Young Sil Eom ${ }^{2 \dagger}$, Jeonggeun Moon ${ }^{1}$, Ho-Jun Jang ${ }^{3}$, Tae-Hoon Kim ${ }^{3}$, Jon Suh ${ }^{4}$, Min Gyu Kong ${ }^{4}$, Sang-Don Park ${ }^{5}$, Sung Woo Kwon ${ }^{5}$, Soon Yong Suh', Kyounghoon Lee', Seung Hwan Han ${ }^{1}$, Taehoon $\mathrm{Ahn}^{1}$ and Woong Chol Kang ${ }^{1 *}$ (1)

\begin{abstract}
Background: Several biomarkers have been proposed as independent predictors of poor outcomes in ST-segment elevation myocardial infarction (STEMI). We investigated whether adding information obtained from routine blood tests including hypoxic liver injury (HLI), dysglycemia, anemia, and high neutrophil to lymphocyte ratio (NLR) could improve the prognostic performance of the TIMI risk score for the prediction of 1-year mortality.

Methods: A total of 1057 patients with STEMI undergoing primary percutaneous coronary intervention (PCI) between 2007 and 2014 were retrospectively enrolled from 4-regional hospitals. HLI and dysglycemia were defined as serum transaminase $>$ twice the normal upper limit and glucose $<90$ or $>250 \mathrm{mg} / \mathrm{dL}$, respectively. The effect of adding biomarkers to the TIMI risk score on its discriminative ability was assessed using c-statistic, net reclassification improvement (NRI), and integrated discrimination improvement (IDI).

Results: The 1-year mortality rate was $7.1 \%$. The best cutoff value of NLR for the prediction of 1-year mortality was 4.3 (sensitivity, 67\%; specificity, 65\%). HLI (HR 2.019; 95\% Cl 1.104-3.695), dysglycemia (HR 2.535; 95\% Cl 1.324-3.923), anemia (HR 2.071; 95\% Cl 1.093-3.923), and high NLR (HR 3.651; 95\% Cl 1.927-6.918) were independent predictors of 1-year mortality. When these 4 parameters were added to the TIMI risk score, the c-statistic significantly improved from 0.841 to $0.876(p<0.001)$, and the NRI and IDI were estimated at $0.203(95 \% \mathrm{Cl} 0.130-0.275 ; p<0.001)$ and 0.089 (95\% Cl 0.060-0.119; $p<0.001)$, respectively.
\end{abstract}

Conclusions: The addition of HLI, dysglycemia, anemia, and high NLR to the TIMI risk score may be useful for very early risk stratification in patients with STEMI receiving primary $\mathrm{PCl}$.

Keywords: ST-segment elevation myocardial infarction, Hypoxic liver injury, Dysglycemia, Anemia, Neutrophil to lymphocyte ratio, Risk score, Mortality

\section{*Correspondence: kangwch@gilhospital.com}

${ }^{\dagger}$ Pyung Chun Oh and Young Sil Eom contributed equally to this work 1 Department of Cardiology, Gil Medical Center, Gachon University College of Medicine, 1198 Guwol-dong, Namdong-gu, Incheon, Republic of Korea 405-760

Full list of author information is available at the end of the article

\section{Background}

Several risk stratification scores have been designed to predict mortality or complications in patients with non-ST-segment elevation myocardial infarction (NSTEMI) and ST-segment elevation myocardial infarction (STEMI). Recent guidelines recommend using risk

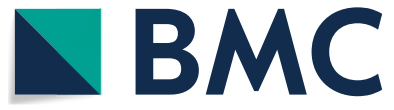

(c) The Author(s) 2020. This article is licensed under a Creative Commons Attribution 4.0 International License, which permits use, sharing, adaptation, distribution and reproduction in any medium or format, as long as you give appropriate credit to the original author(s) and the source, provide a link to the Creative Commons licence, and indicate if changes were made. The images or other third party material in this article are included in the article's Creative Commons licence, unless indicated otherwise in a credit line to the material. If material is not included in the article's Creative Commons licence and your intended use is not permitted by statutory regulation or exceeds the permitted use, you will need to obtain permission directly from the copyright holder. To view a copy of this licence, visit http://creativecommons.org/licenses/by/4.0/. The Creative Commons Public Domain Dedication waiver (http://creativecommons.org/publicdomain/zero/1.0/) applies to the data made available in this article, unless otherwise stated in a credit line to the data. 
scores, for early risk assessment and adjustment, such as the TIMI (Thrombolysis in Myocardial Infarction) for STEMI, TIMI for NSTEMI, and GRACE (Global Registry of Acute Coronary Events) for both types of myocardial infarction (MI) [1, 2].

Recent studies suggest that several biomarkers evaluated during routine blood tests upon admission may be independent predictors of poor clinical outcomes in patients with STEMI [3-5]. We previously demonstrated that the presence of hypoxic liver injury (HLI), defined as serum transaminase levels more than two times of the upper normal limit, was associated with all-cause mortality and major adverse cardiovascular events in STEMI patients [5]. Dysglycemia, defined as serum glucose $<90$ or $>250 \mathrm{mg} / \mathrm{dL}$ at the time of presentation, was an independent predictor of in-hospital mortality in STEMI [6]. In addition, the neutrophil to lymphocyte ratio (NLR) has been demonstrated as a prognostic biomarker for various cardiovascular diseases [7, 8]. Several studies have revealed that STEMI patients with anemia had a higher risk of cardiovascular mortality $[3,9]$. However, there was little evidence that examining these biomarkers provided any incremental value to conventional risk scoring models. Therefore, we investigated whether the addition of HLI, dysglycemia, NLR, and anemia, measured upon on admission, could improve the prognostic performance of the TIMI risk score for 1-year mortality in patients with STEMI who underwent primary percutaneous coronary intervention (PCI).

\section{Methods}

\section{Study population}

We retrospectively analyzed the cohort data from INTERSTELLAR (INcheon-Bucheon cohorT of patients undERgoing primary PCI for acute ST-ELevation myocardiaL infarction; clinicaltrials.gov identifier NCT02804958) $[10,11]$. The INTERSTELLAR registry was retrospectively established by four hospitals in the Incheon-Bucheon province. This cohort consisted of 1540 consecutive patients with STEMI who underwent primary PCI between 2007 and 2014. The study protocol was approved by the Institutional Review Boards of the four participating hospitals and written informed consent was obtained from each patient. In this cohort, patients were excluded if they had any one of the following; primary cardiomyopathy, significant valvular heart disease ( $\geq$ moderate), pericardial disease, known liver disease, or history of potentially hepatotoxic medications within the past 3 months. Among these, all 483 patients from one hospital were excluded because their baseline laboratory data, particularly the NLR, was incomplete. Thus, 1057 patients from the remaining three hospitals were enrolled in this analysis. All procedures and in-hospital management were performed according to current standard guidelines, as previously described [11, 12].

\section{Definition of variables and endpoint}

HLI was defined as serum transaminase levels of more than two times the normal upper limit (i.e., aspartate transaminase $>80 \mathrm{U} / \mathrm{L}$ or alanine transaminase $>80 \mathrm{U} / \mathrm{L}$ ), as per Moon et al. [5]. We defined dysglycemia as serum glucose $<90$ or $>250 \mathrm{mg} / \mathrm{dL}$, based on our previous study using the same cohort [6]. In our previous study [6], the median serum glucose level of the overall population was $144 \mathrm{mg} / \mathrm{dL}$. Among study subjects with serum glucose $<140 \mathrm{mg} / \mathrm{dL}$ in emergency room, glucose $<89.5 \mathrm{mg} /$ $\mathrm{dL}$ was a highly specific predictor of in-hospital death (sensitivity 25\%; specificity 97\%; area under the curve [AUC] 0.683; $p=0.005)$. Similarly, among patients with serum glucose $>140 \mathrm{mg} / \mathrm{dL}$, a cut-off value of $241.5 \mathrm{mg} /$ $\mathrm{dL}$ predicted in-hospital death (sensitivity, $60 \%$; specificity, 74\%; AUC 0.706; $p<0.001$ ). According to the World Health Organization classification, anemia was defined as a hemoglobin level of $<12.0 \mathrm{~g} / \mathrm{dL}$ in women and $<13.0 \mathrm{~g} /$ $\mathrm{dL}$ in men. The best cut-off value for NLR was statistically calculated. The primary endpoint was cumulative all-cause death in 1 year. All medical records were reviewed to collect patient follow-up data. In the case of follow-up failure, standardized telephone interviews were conducted.

\section{Statistical analysis}

Continuous data are presented as means \pm standard deviations and/or medians (interquartile ranges), as appropriate. The categorical variables are described using percentage or absolute number. Baseline characteristics were compared across patients with and without 1-year all-cause mortality. A receiver operating characteristics (ROC) curve was calculated to determine the cut-off value of NLR for predicting 1-year mortality. The cut-off value for optimal sensitivity and specificity, together with the AUC, was also identified. Multivariate Cox regression analysis was performed to identify independent predictors of primary endpoint. In the full regression model, the hazard ratio for each of the additional blood biomarkers was calculated. For each patient, additional risk scores were calculated as the simple arithmetic sum of point values assigned to each risk factor based on the multivariate-adjusted risk relationship: 1 point for hazard ratio (HR) $1.0-<2.5$, and 2 points for $H R>2.5$. The discriminative ability and prognostic performance of the combination of TIMI risk score and blood biomarkers, as compared to the TIMI risk score alone, was assessed by C-statistics, integrated discrimination index (IDI), and net 
reclassification index (NRI). Risk categories for mortality were set at $<1 \%$ for low risk, $1 \%-5 \%$ for intermediate risk and $>5 \%$ for high risk. The calibration of models was assessed by the Hosmer and Lemeshow test. No adjustments for multiple testing were performed as this was an exploratory analysis. P values of less than 0.05 were considered statistically significant. The analysis was performed using SAS version 9.4 (SAS Institute, Cary, NC, USA).

\section{Results}

Baseline characteristics

The mean age was $60.5 \pm 13.1$ years, and $79.2 \%$ of the patients were men. Median duration of follow-up was 27 months (interquartile range, 10-41 months). Oneyear mortality was seen in 75 patients (7.1\%). Table 1 shows the demographic and laboratory data measured at the time of presentation and intergroup comparison between the groups with or without 1-year mortality,

Table 1 Demographic and laboratory data

\begin{tabular}{|c|c|c|c|c|}
\hline & All $(n=1057)$ & Mortality $(-)(n=982)$ & Mortality $(+)(n=75)$ & $p$ value \\
\hline \multicolumn{5}{|l|}{ Demographic data } \\
\hline Age (years) & $60.5 \pm 13.1$ & $59.7 \pm 12.9$ & $70.8 \pm 11.0$ & $<0.001$ \\
\hline Men $[n(\%)]$ & $837(79.2)$ & $784(79.8)$ & $53(70.7)$ & 0.075 \\
\hline Body mass index $\left(\mathrm{kg} / \mathrm{m}^{2}\right)$ & $24.1 \pm 3.2$ & $24.2 \pm 3.2$ & $23.0 \pm 3.6$ & 0.002 \\
\hline Diabetes mellitus [n (\%)] & $279(26.4)$ & $250(25.5)$ & $29(38.7)$ & 0.020 \\
\hline Hypertension [n (\%)] & $496(46.9)$ & $452(46.0)$ & $44(58.7)$ & 0.041 \\
\hline Systolic blood pressure (mmHg) & $125.1 \pm 27.3$ & $126.3 \pm 26.7$ & $109.0 \pm 30.8$ & $<0.001$ \\
\hline Diastolic blood pressure $(\mathrm{mmHg})$ & $76.3 \pm 17.5$ & $77.0 \pm 17.2$ & $66.9 \pm 19.2$ & $<0.001$ \\
\hline Heart rate (beats/minute) & $77.5 \pm 20.1$ & $76.7 \pm 19.5$ & $87.3 \pm 25.0$ & 0.001 \\
\hline Cardiogenic shock [n (\%)] & $66(6.2)$ & $47(4.8)$ & $19(25.3)$ & $<0.001$ \\
\hline Killip class II-IV [n (\%)] & $209(19.8)$ & $168(17.1)$ & $41(54.7)$ & $<0.001$ \\
\hline Anterior wall infarction [n (\%)] & $564(69.3)$ & $512(52.1)$ & $52(69.3)$ & 0.004 \\
\hline \multicolumn{5}{|l|}{ Laboratory data } \\
\hline Albumin (g/dL) & $4.2 \pm 0.4$ & $4.2 \pm 0.4$ & $3.8 \pm 0.6$ & $<0.001$ \\
\hline Glucose (mg/dL) & $177.7 \pm 83.1$ & $173.0 \pm 74.6$ & $239.4 \pm 144.0$ & $<0.001$ \\
\hline Dysglycemia [n (\%)] & $159(15.0)$ & $126(12.8)$ & $33(44.0)$ & $<0.001$ \\
\hline Total bilirubin (mg/dL) & $0.7 \pm 0.4$ & $0.7 \pm 0.4$ & $0.7 \pm 0.5$ & 0.245 \\
\hline AST $(I U / L)$ & $33.0(23.0-65.0)$ & $32.0(23.0-61.0)$ & $72.0(27.0-227.0)$ & $<0.001$ \\
\hline $\mathrm{ALT}(\mathrm{IU} / \mathrm{L})$ & $26.0(19.0-42.0)$ & $26.0(19.0-41.0)$ & $36.0(18.0-78.0)$ & 0.034 \\
\hline Hypoxic liver injury ${ }^{\dagger}[n(\%)]$ & $236(22.3)$ & $199(20.3)$ & $37(49.3)$ & $<0.001$ \\
\hline$A L P(I \cup / L)$ & $74.0(61.0-91.1)$ & $74.0(61.0-90.0)$ & $81.0(65.0-98.0)$ & 0.061 \\
\hline Creatinine $(\mathrm{mg} / \mathrm{dL})$ & $1.08 \pm 0.73$ & $1.06 \pm 0.73$ & $1.33 \pm 0.67$ & 0.001 \\
\hline Estimated GFR $\left(\mathrm{mL} / \mathrm{min} / 1.73 \mathrm{~m}^{2}\right)$ & $82.1 \pm 27.5$ & $83.3 \pm 26.8$ & $65.8 \pm 32.0$ & $<0.001$ \\
\hline Estimated GFR $<60 \mathrm{~mL} / \mathrm{min} / 1.73 \mathrm{~m}^{2}[\mathrm{n}(\%)]$ & $184(17.4)$ & $152(15.5)$ & $32(42.7)$ & $<0.001$ \\
\hline Hemoglobin (mg/dL) & $14.2 \pm 1.9$ & $14.3 \pm 1.9$ & $13.1 \pm 2.4$ & $<0.001$ \\
\hline Anemia [n (\%)] & $177(16.7)$ & $146(14.9)$ & $31(41.3)$ & $<0.001$ \\
\hline $\mathrm{WBC}\left(\times 10^{3} / \mu \mathrm{L}\right)$ & $12.0 \pm 5.2$ & $11.8 \pm 5.2$ & $13.9 \pm 4.7$ & 0.001 \\
\hline NLR & $3.1(1.6-5.7)$ & $3.0(1.5-5.4)$ & $5.4(3.0-8.9)$ & $<0.001$ \\
\hline$N L R>4.3[n(\%)]$ & $393(37.2)$ & $342(34.8)$ & $51(68.0)$ & $<0.001$ \\
\hline Total cholesterol (mg/dL) & $189.3 \pm 43.7$ & $190.6 \pm 43.1$ & $172.5 \pm 47.8$ & 0.001 \\
\hline LDL-cholesterol (mg/dL) & $117.0 \pm 36.8$ & $117.8 \pm 36.7$ & $106.6 \pm 37.0$ & 0.043 \\
\hline HDL-cholesterol (mg/dL) & $42.5 \pm 11.0$ & $42.6 \pm 10.9$ & $41.0 \pm 11.7$ & 0.305 \\
\hline Triglyceride & $129.0(86.0-196.0)$ & $131.0(87.0-197.0)$ & $111.0(76.5-180.5)$ & 0.100 \\
\hline Initial CK-MB (ng/mL) & $5.3(2.1-26.5)$ & $4.9(2.1-22.5)$ & $26.0(3.6-96.1)$ & $<0.001$ \\
\hline Peak CK-MB (ng/mL) & $177.4(78.2-300.0)$ & $170.9(77.8-300.0)$ & $271.0(86.0-332.2)$ & 0.016 \\
\hline
\end{tabular}

AST aspartate transaminase, $A L T$ alanine transaminase, $A L P$ alkaline phosphatase, GFR glomerular filtration rate, WBC white blood cell, NLR neutrophil to lymphocyte ratio, $C K-M B$ creatine kinase-myocardial band isoenzyme

* Dysglycemia was defined as serum glucose $<90$ or $>250 \mathrm{mg} / \mathrm{dL}$

${ }^{\dagger}$ Hypoxic liver injury was defined as an elevation of serum transaminases levels more than twice the upper limit of normal 
defined henceforth as Mortality (+) or Mortality (-), respectively. Mortality $(+)$ patients were significantly older and had lower blood pressure, higher pulse rate, lower body mass index, higher Killip class, and higher prevalence of anterior wall infarction. Regarding the laboratory findings, the Mortality $(+)$ group showed significantly higher serum transaminases, lower albumin, higher glucose, lower estimated glomerular filtration rate, lower hemoglobin, higher white blood cell counts, and higher NLR. The HLI, dysglycemia, and anemia were significantly higher in the Mortality $(+)$ group than in the Mortality ( - ) group (49.3 vs $20.3 \%$ for HLI, 44.0 vs. $12.8 \%$ for dysglycemia, 41.3 vs. $14.9 \%$ for anemia, respectively, all $p<0.001$ ). For predicting 1-year mortality, the cut-off value of NLR was determined to be 4.3 , with a sensitivity and specificity of $66.7 \%$ and $65.2 \%$, respectively (AUC $0.681 ; 95 \%$ CI $0.614-0.749, p<0.001)$. High NLR values $(>4.3)$ were more frequent in the Mortality $(+)$ group than those in the Mortality (-) group (68.0 vs $34.8 \%$, respectively, $p<0.001)$.
Angiographic, procedural, and echocardiographic data is summarized in Table 2. Although baseline TIMI flow grade was similar between the two groups, the final TIMI flow grades and procedural success rates were significantly lower in the Mortality (+) group. The Mortality $(+)$ group also had longer door-to-balloon time and symptom-to-balloon time. Further, the left ventricular ejection fraction was significantly lower in the Mortality $(+)$ group than that in the Mortality ( - ) group (35.7 vs. $48.0 \%$, respectively, $p<0.001$ ).

\section{Predictors of 1-year mortality}

In the multivariate Cox regression analysis, age, systolic blood pressure $<100 \mathrm{mmHg}$, Killp class II-IV, left ventricular ejection fraction $<40 \%$, and anterior wall infarction were significant and were independent predictors for 1-year mortality (Table 3). Among the TIMI risk score variables, history of diabetes mellitus, hypertension, or angina, heart rate, body weight, and ischemia time were not significant risk factors in the multivariate analysis. However, HLI, dysglycemia, anemia, and high NLR were

Table 2 Angiographic, procedural and echocardiographic data

\begin{tabular}{|c|c|c|c|c|}
\hline & All $(n=1057)$ & Mortality $(-)(n=982)$ & Mortality $(+)(n=75)$ & $p$ value \\
\hline \multicolumn{5}{|l|}{ Angiographic and procedural data } \\
\hline Infarct related artery [n (\%)] & & & & NA \\
\hline Left main & $8(0.8)$ & $2(0.2)$ & $6(8.0)$ & \\
\hline Left anterior descending & $556(52.6)$ & $510(51.9)$ & $46(61.3)$ & \\
\hline Left circumflex & $95(9.0)$ & $90(9.2)$ & $5(6.7)$ & \\
\hline Right coronary & $398(37.7)$ & $380(38.7)$ & $18(24.0)$ & \\
\hline \multicolumn{2}{|c|}{ Extent of coronary artery disease [n (\%)] } & & & 0.257 \\
\hline 1-vessel & $404(38.2)$ & $382(38.9)$ & $22(29.3)$ & \\
\hline 2-vessel & $364(34.4)$ & $334(34.0)$ & $30(40.0)$ & \\
\hline 3-vessel & $289(27.3)$ & $266(27.1)$ & $23(30.7)$ & \\
\hline \multicolumn{2}{|l|}{ Baseline TIMI flow grade [n (\%)] } & & & 0.840 \\
\hline $0-2$ & $950(90.0)$ & $884(90.1)$ & $66(89.2)$ & \\
\hline 3 & $105(10.0)$ & $97(9.9)$ & $8(10.8)$ & \\
\hline \multicolumn{2}{|l|}{ Final TIMI flow grade [n (\%)] } & & & $<0.001$ \\
\hline $0-2$ & $163(15.5)$ & $134(13.7)$ & $29(39.2)$ & \\
\hline 3 & $892(84.5)$ & $847(86.3)$ & $45(60.8)$ & \\
\hline Stent number $(\mathrm{n})$ & $1.1 \pm 0.3$ & $1.1 \pm 0.3$ & $1.2 \pm 0.5$ & 0.036 \\
\hline Stent diameter (mm) & $3.09 \pm 0.38$ & $3.10 \pm 0.38$ & $2.95 \pm 0.40$ & 0.002 \\
\hline Stent length (mm) & $27.1 \pm 10.5$ & $26.8 \pm 10.3$ & $31.0 \pm 13.3$ & 0.012 \\
\hline Door-to-balloon time (min) & $72.0(58.0-87.0)$ & $71.0(58.0-87.0)$ & $80.0(64.5-89.0)$ & 0.012 \\
\hline Symptom-to-balloon time (min) & $210.0(135.0-407.5)$ & $206.0(133.0-381.3)$ & $296.0(180.0-570.0)$ & 0.001 \\
\hline Procedural success [n (\%)] & $892(84.5)$ & $847(86.3)$ & $45(60.8)$ & $<0.001$ \\
\hline \multicolumn{5}{|l|}{ Echocardiographic data } \\
\hline $\operatorname{LVEF}(\%)$ & $47.3 \pm 12.0$ & $48.0 \pm 11.5$ & $35.7 \pm 14.9$ & $<0.001$ \\
\hline $\operatorname{LVEDD~(mm)~}$ & $51.4 \pm 5.0$ & $51.4 \pm 5.0$ & $51.2 \pm 4.8$ & 0.897 \\
\hline$E / E^{\prime}$ & $11.9 \pm 5.2$ & $11.8 \pm 5.2$ & $12.8 \pm 4.7$ & 0.564 \\
\hline
\end{tabular}

NA not available, TIMI thrombolysis in myocardial infarction, LVEF left ventricular ejection fraction, LVEDD left ventricular end-diastolic dimension 
Table 3 Predictors for 1-year all-cause death

\begin{tabular}{|c|c|c|c|c|c|c|}
\hline \multirow[t]{2}{*}{ Variables } & \multicolumn{3}{|c|}{ Univariate analysis } & \multicolumn{3}{|c|}{ Multivariate analysis } \\
\hline & HR & $95 \% \mathrm{Cl}$ & $p$ value & HR & $95 \% \mathrm{Cl}$ & $p$ value \\
\hline Age (per 10-year-old increase) & 1.960 & $1.621-2.370$ & $<0.001$ & 1.416 & $1.097-1.828$ & 0.008 \\
\hline Male & 0.610 & $0.371-1.003$ & 0.051 & 1.783 & $0.802-3.962$ & 0.156 \\
\hline Body weight $<67$ kg & 1.807 & $1.123-2.907$ & 0.015 & 1.555 & $0.735-3.291$ & 0.249 \\
\hline Diabetes mellitus & 1.824 & $1.146-2.903$ & 0.011 & 1.062 & $0.496-2.272$ & 0.878 \\
\hline Hypertension & 1.655 & $1.045-2.621$ & 0.032 & 0.970 & $0.489-1.923$ & 0.930 \\
\hline Systolic BP $<100$ mmHg & 4.459 & $2.770-7.178$ & $<0.001$ & 2.568 & $1.357-4.860$ & 0.004 \\
\hline Heart rate $>100$ beats/minute & 3.022 & $1.796-5.085$ & $<0.001$ & 1.239 & $0.548-2.802$ & 0.606 \\
\hline Killip class II-IV & 5.445 & $3.455-8.581$ & $<0.001$ & 2.426 & $1.367-4.303$ & 0.002 \\
\hline LVEF $<40 \%$ & 5.080 & $2.981-8.658$ & $<0.001$ & 1.889 & $1.008-3.541$ & 0.047 \\
\hline Anterior wall infarction & 2.061 & $1.261-3.366$ & 0.004 & 2.084 & $1.056-4.114$ & 0.034 \\
\hline Multivessel disease & 1.526 & $0.928-2.509$ & 0.095 & 1.047 & $0.511-2.144$ & 0.900 \\
\hline Post-TIMI flow $<3$ & 3.782 & $2.371-6.032$ & $<0.001$ & 1.843 & $1.006-3.379$ & 0.048 \\
\hline Hypoxic liver injury* & 3.631 & $2.308-5.710$ & $<0.001$ & 2.019 & $1.104-3.695$ & 0.023 \\
\hline Dysglycemia $^{\dagger}$ & 5.008 & $3.173-7.904$ & $<0.001$ & 2.535 & $1.324-4.855$ & 0.005 \\
\hline Anemia & 3.702 & $2.338-5.863$ & $<0.001$ & 2.071 & $1.093-3.923$ & 0.026 \\
\hline$N L R>4.3$ & 3.796 & $2.336-6.166$ & $<0.001$ & 3.651 & $1.927-6.918$ & $<0.001$ \\
\hline Estimated GFR $<60 \mathrm{~mL} / \mathrm{min} / 1.73 \mathrm{~m}^{2}$ & 3.879 & $2.454-6.131$ & $<0.001$ & 0.791 & $0.364-1.720$ & 0.554 \\
\hline Peak CK-MB (log) & 1.637 & $0.951-2.821$ & 0.075 & 1.228 & $0.618-2.441$ & 0.558 \\
\hline Symptom to balloon time $>4 \mathrm{~h}$ & 2.094 & $1.316-3.333$ & 0.002 & 1.477 & $0.731-2.988$ & 0.277 \\
\hline
\end{tabular}

$H R$ hazard ratio, $C l$ confidence interval, $B P$ blood pressure, $L V E F$ left ventricular ejection fraction, NLR neutrophil to lymphocyte ratio, GFR glomerular filtration rate, $C K$ $M B$ creatine kinase-myocardial band isoenzyme, $A L P$ alkaline phosphatase

* Hypoxic liver injury was defined as an elevation of serum transaminase level more than twice the upper limit of normal

+ Dysglycemia was defined as serum glucose $<90$ or $>250 \mathrm{mg} / \mathrm{dL}$

all independently associated with increased risk of 1 -year mortality.

\section{Prognostic value of HLI, dysglycemia, NLR, and anemia over the TIMI risk score}

The overall discriminatory ability in predicting the 1-year mortality was reasonable for TIMI risk score (c-statistic 0.841; 95\% CI 0.796-0.886). The addition of HLI, dysglycemia, NLR, and anemia to the TIMI risk score significantly improved the c-statistic (from 0.841 to 0.876 , $p<0.001$; Fig. 1). The incremental improvement of the c-statistics was confirmed in each subgroup with Killip class I and class II-IV (Additional file 1: Figure S1). Table 4 shows reclassification for patients who died and those who did not, across 3 strata risks for 1-year mortality. When incorporating blood biomarkers, the risk reclassification was more accurate in $265(27.0 \%)$ and less accurate in $66(6.7 \%)$ patients, among a total of 982 patients of the Mortality (-) group (net improvement, 20.3\%). However, addition of blood biomarkers to the TIMI risk score did not improve the reclassification of the Mortality $(+)$ group (net improvement, $0.0 \%$ ). Overall the NRI and IDI for predicting 1-year mortality using blood biomarkers plus TIMI risk score were 0.203 (95\%
CI $0.130-0.275 ; p<0.001)$ and $0.089(95 \%$ CI 0.060 $0.119 ; p<0.001)$, respectively.

\section{Discussion}

The main findings of this study include: (1) HLI, dysglycemia, high NLR, and anemia were independently associated with the risk of 1-year mortality after primary PCI in patients with STEMI and (2) addition of these blood biomarkers to the TIMI risk score for STEMI significantly improved the discriminatory performance. To our knowledge, this is the first study to report that integrating blood biomarkers routinely measured in the emergency room with a conventional risk score incrementally improves the accuracy of 1 -year mortality prediction in STEMI.

Many biomarkers are routinely measured at the time of presentation to monitor the general health status of a patient, including liver function, glucose metabolism, presence of anemia, or inflammation status [13]. These biomarkers are not commonly used for risk stratification in STEMI. In addition, conventional risk scoring systems for STEMI do not include routinely measured biomarkers as predictors, except for serum creatinine or hemoglobin. Previous studies have revealed that many 


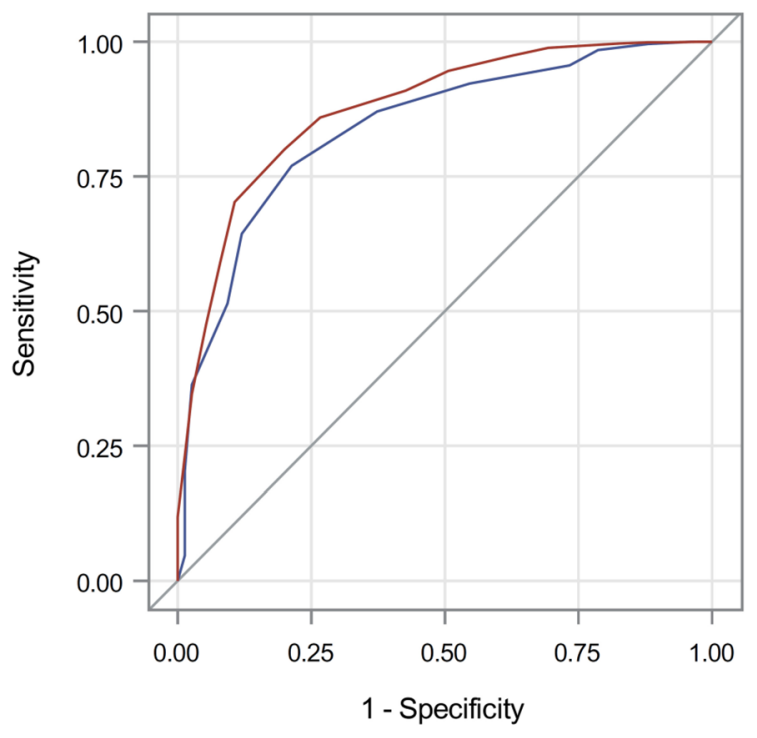

TIMI risk score

TIMI risk score + blood biomarkers ${ }^{\dagger}$

\begin{tabular}{lccc}
\hline Risk score & C-Statistic & $95 \% \mathrm{Cl}$ & p value \\
\hline TIMI & 0.8408 & $0.7959-0.8857$ & \\
TIMI + blood biomarkers ${ }^{\dagger}$ & 0.8760 & $0.8356-0.9164$ & 0.0009 \\
\hline
\end{tabular}

Fig. 1 Receiver operating curves for the predicted probabilities of selected risk scores before (blue line) and after (red line) the addition of four biomarkers to the conventional TIMI risk score. ${ }^{\dagger}$ The added biomarkers included hypoxic liver injury, dysglycemia, anemia and high neutrophil to lymphocyte ratio

Table 4 Reclassification among patient who died and those who did not die at 1-year after ST-segment elevation myocardial infarction using the routinely measured blood biomarkers over the TIMI risk score

\begin{tabular}{|c|c|c|c|c|}
\hline \multirow[t]{2}{*}{ 1-year mortality } & \multicolumn{4}{|c|}{ Model using blood biomarkers ${ }^{\dagger}$ plus TIMI risk score } \\
\hline & Low risk ( $<1 \%)$ & Moderate risk (1-5\%) & High risk (>5\%) & Total \\
\hline \multicolumn{5}{|c|}{ Model using TIMI risk score } \\
\hline \multicolumn{5}{|l|}{ No events } \\
\hline Low risk (<1\%) & $176(88.44)$ & $23(11.56)$ & $0(0)$ & 199 \\
\hline Moderate risk (1-5\%) & $164(37.88)$ & $226(52.19)$ & $43(9.93)$ & 4331 \\
\hline High risk (>5\%) & $0(0)$ & $101(28.86)$ & $249(71.14)$ & 350 \\
\hline Total & 340 & 350 & 292 & 982 \\
\hline \multicolumn{5}{|l|}{ Events } \\
\hline Low risk (<1\%) & $1(100.0)$ & $0(0)$ & $0(0)$ & 1 \\
\hline Moderate risk (1-5\%) & $1(12.50)$ & $4(50.0)$ & $3(37.50)$ & 8 \\
\hline High risk (>5\%) & $0(0)$ & $2(3.03)$ & $64(96.97)$ & 66 \\
\hline Total & 2 & 6 & 67 & 75 \\
\hline \multicolumn{5}{|c|}{$\begin{array}{l}N R \mid=0.203(95 \% \mathrm{Cl} 0.130-0.275) \\
\mid \mathrm{DI}=0.089(95 \% \mathrm{Cl} 0.060-0.119)\end{array}$} \\
\hline
\end{tabular}

$N R I$ net reclassification improvement, IDI integrated discrimination improvement

† The blood biomarkers included hypoxic liver injury, dysglycemia, anemia, and high neutrophil to lymphocyte ratio

biomarkers, such as serum transaminases, glucose, or NLR, are independently associated with poor outcomes in STEMI $[3,5,6,9,14,15]$. However, there is little data that comprehensively evaluates the prognostic impacts of routinely measured blood biomarkers and the incremental value of combining these with a conventional 
risk score. In this study, we tried to integrate routine blood biomarkers for early risk stratification in STEMI patients. Early in the study, we considered serum creatinine, alkaline phosphatase, mean platelet volume, and creatine kinase-myocardial band isoenzyme as prognosticator. However, these biomarkers were not independent predictors for 1-year mortality. Finally, we included four biomarkers including HLI, dysglycemia, anemia, and high NLR among routinely measured blood tests on admission. In this respect, our model, which adds four predictors (HLI, dysglycemia, high NLR, and anemia) determined from routine blood tests on admission to an established TIMI risk score, had a substantial incremental impact on the discriminatory power. In particular, our combined model improved risk reclassification in patients without mortality, suggesting a more accurate capacity to reliably identify patients with low risk for mortality. This may help to select low-risk patients for early hospital discharge. A study of a prospective cohort, consisting of nearly 2000 MI patients, reported that adding several routine biomarkers returned a significantly better prediction of mortality or recurrent MI within 6 months than the GRACE risk score [16]. This study used 9 biomarkers as continuous variables, including urea, sodium, potassium, alkaline phosphatase, lowdensity lipoprotein cholesterol, glucose, hemoglobin, and C-reactive protein. It is difficult in practice to apply an additional biomarker as a continuous variable to a conventional risk scoring system at the bedside. Therefore, we divided biomarkers into binary variables, according to cut-off values that were previously determined or statistically calculated. This is a more practical approach for risk stratification at the time of presentation, without the aid of a computer. Yanish et al. [17] also developed a laboratory stratification model from the combination of routine blood tests to predict in-hospital mortality in patients with STEMI. Five variables (white blood cell, hemoglobin, C-reactive protein, creatinine, and glucose) were included in the laboratory model. The performance of this model was comparable to that of TIMI risk score and could be helpful in predicting in-hospital prognosis of STEMI patients by further subdividing the TIMI risk score. There are some differences between Yanish et al.s study and ours. We focused 1-year mortality as an endpoint and integrated routine blood biomarkers into TIMI risk score, just not subclassifying the conventional risk score. In addition, HLI and NLR, recently emerged as prognostic biomarkers, included for our risk model.

It has been previously reported that each individual component of our risk model is significantly related with a high risk of adverse events. Recently, there have been a few reports stating the independent association between serum transaminases and cardiovascular outcomes in patients with STEMI $[5,18,19]$. The HLI, defined as increase in serum transaminases more than two times the upper limit of normal, was associated with high risk of mortality and major adverse cardiovascular events after primary PCI in STEMI [5]. Of note, Moon et al. [5] reported that HLI was closely correlated with left ventricular systolic dysfunction after PCI. Furthermore, admission and peak levels of serum transaminases were significantly related with infarct size, left ventricular ejection fraction, and the presence of microvascular damage in a study using cardiac magnetic resonance imaging [19]. The presence of HLI on admission may be a very sensitive marker suggesting significant left ventricular systolic dysfunction before primary PCI.

Previous studies have described the relationship between hyperglycemia on admission and poor outcomes in acute MI, regardless of the diabetic status [14, 20]. Hyperglycemia might aggravate organ damage by inflammation, endothelial dysfunction, increasing oxidative stress, and coagulation [21-23]. Hypoglycemia on admission has also been related to higher mortality, suggesting a U-shaped relationship between glucose levels and poor prognosis [15]. A plausible mechanism is that hypoglycemia increases the risk of arrhythmia, through changes in autonomic activity and effects on repolarization [24]. Our group also reported that dysglycemia with the same definition as that of the present study, was an independent predictor of in-hospital death in STEMI [6].

Anemia is associated with an increased risk for morbidity and mortality in acute MI [3,9]. Anemia can potentially worsen myocardial ischemic insult by decreasing the oxygen content of blood delivered to the stressed heart and increasing myocardial oxygen demand and cardiac output for adequate systemic oxygen perfusion $[25,26]$. Anemic patients have a higher prevalence of baseline comorbidities and receive less guideline-based pharmacological treatments and reperfusion therapy than non-anemic patients [27]. Anemia on admission is also associated with an increased risk of contrast-induced nephropathy after primary PCI [28]. Among the established risk scoring models in STEMI, only the CADILLAC (the Controlled Abciximab and Device Investigation to Lower Late Angioplasty Complications) risk score includes anemia as a predictor of mortality [29].

Finally, the NLR has recently emerged as a potential prognostic biomarker for various cardiovascular diseases $[7,8]$. A high NLR has been associated with an increased risk of short- and long-term mortality after primary PCI in patients with STEMI [30,31]. The NLR is an indicator of systemic inflammation, which plays a role in the repair and remodeling of infarcted heart tissue [32]. A high NLR $(>4.25)$ could predict adverse left ventricular remodeling 6 months after anterior STEMI [33]. Among patients 
with acute MI who underwent PCI, a high post-PCI NLR was related with the extent of myocardial infarction, as measured by cardiac magnetic resonance imaging [4]. In the present study, the best cut-off value of NLR to predict 1 -year mortality was 4.3 , which was consistent with that in previous studies [30,31]. A high NLR was the most powerful predictor of 1-year mortality among the biomarkers routinely measured in blood tests.

The combination of HLI, anemia, dysglycemia, and NLR may represent an additional biochemical marker for indicating the severity and extent of myocardial damage, as well as the presence of adverse cardiac remodeling. This information can enhance other conventional risk predictors, such as clinical presentation, left ventricular systolic function, and atherosclerotic burden. The information on HLI, anemia, dysglycemia and NLR is typically taken from the very first routine blood test at the time of presentation, even before PCI. They are readily available and not costly. Thus, our study suggests that the combination of HLI, anemia, dysglycemia, and NLR with a traditional risk score model may provide valuable information for very early risk stratification in patients with STEMI undergoing primary PCI.

There are several limitations in this study. First, as an observational study, it is possible that residual confounding factors not adjusted by multivariate analysis were partially responsible for the present findings. Second, it is not conclusive that elevated serum transaminases were solely associated with hypoxic liver damage. In addition, the cut-off value of HLI have not been fully verified. Third, we had to calculate the best cut-off value of NLR, which may raise statistical inaccuracy. Fourth, the cut-off value of dysglycemia was determined based on our previous study, which was not validated in an external cohort. Finally, we did not externally validate our findings. Further studies are required to validate our findings externally and prospectively.

\section{Conclusions}

The addition of routine blood biomarkers including HLI, dysglycemia, anemia, and high NLR to the TIMI risk score for STEMI significantly improved the discriminatory performance to predict 1-year mortality. Therefore, these biomarkers may be useful for very early risk stratification in patients with STEMI receiving primary PCI.

\section{Supplementary information}

Supplementary information accompanies this paper at https://doi. org/10.1186/s12872-020-01777-7.

Additional file 1. Fig. S1. Receiver operating curves for the predicted probabilities of selected risk scores before (blue line) and after (red line) the addition of four biomarkers to the conventional TIMI risk score in each subgroup with Killip class I (A) and class II-IV (B). TThe added biomarkers included hypoxic liver injury, dysglycemia, anemia and high neutrophil to lymphocyte ratio.

\section{Abbreviations}

NSTEMI: Non-ST-segment elevation myocardial infarction; STEMI: ST-segment elevation myocardial infarction; TIMI: Thrombolysis in myocardial infarction; GRACE: Global Registry of Acute Coronary Events; MI: Myocardial infarction; HLI: Hypoxic liver injury; NLR: Neutrophil to lymphocyte ratio; PCI: Percutaneous coronary intervention; ROC: Receiver operating characteristics; AUC: Area under the curve; HR: Hazard ratio; IDI: Integrated discrimination index; NRI: Net reclassification index.

\section{Acknowledgements}

None.

\section{Authors' contributions}

PCO, JM and WCK conceived and designed the study. PCO, JM, HJJ, THK, JS, MGK, SDP, SWK, SYS, KL, SHH, and TA collected clinical data and helped to interpret of data. PCO and EYS performed statistical analysis. PCO wrote the paper. EYS and WCK reviewed and substantively edited the manuscript. All authors read and approved the final manuscript.

\section{Funding}

This work was supported by the Yuhan corporation (Seoul, Republic of Korea) and a grant of the Korea Health Technology R\&D Project through the Korea Health Industry Development Institute (KHIDI), funded by the Ministry of Health \& Welfare, Republic of Korea (grant number: HI16C2319). The funder had no role in study design, data collection and analysis, decision to publish, or preparation of the manuscript.

\section{Availability of data and materials}

The datasets used and/or analysed during the current study are available from the corresponding author on reasonable request.

\section{Ethics approval and consent to participate}

The Institutional Review Boards (Gachon University Gil Medical Center Institutional Review Board, Sejong General Hospital Institutional Review Board, Soon Chun Hyang University Bucheon Hospital Institutional Review Board, and Inha University Hospital Institutional Review Board) of the four participating hospitals approved the study protocol and written informed consent was obtained from each patient. The study protocol conforms to the ethical guidelines of the 1975 Declaration of Helsinki as reflected in a priori approval by the institution's human research committee.

\section{Consent for publication}

Not applicable.

\section{Competing interests}

The authors report no relationships that could be construed as a conflict of interest.

\section{Author details \\ 1 Department of Cardiology, Gil Medical Center, Gachon University College of Medicine, 1198 Guwol-dong, Namdong-gu, Incheon, Republic of Korea 405-760. ${ }^{2}$ Department of Endocrinology and Metabolism, Gil Medical Center, Gachon University College of Medicine, Incheon, Republic of Korea. ${ }^{3}$ Depart- ment of Cardiology, Sejong General Hospital, Bucheon, Republic of Korea. ${ }^{4}$ Department of Cardiology, Soon Chun Hyang University Bucheon Hospital, Bucheon, Republic of Korea. ${ }^{5}$ Department of Cardiology, Inha University Hospital, Incheon, Republic of Korea.}

Received: 10 July 2020 Accepted: 9 November 2020 Published online: 18 November 2020 


\section{References}

1. Amsterdam EA, Wenger NK, Brindis RG, Casey DE Jr, Ganiats TG, Holmes DR Jr, et al. 2014 AHA/ACC guideline for the management of patients with non-ST-elevation acute coronary syndromes: a report of the American College of Cardiology/American Heart Association Task Force on Practice Guidelines. J Am Coll Cardiol. 2014;64(24):e139-228.

2. O'Gara PT, Kushner FG, Ascheim DD, Casey DE Jr, Chung MK, de Lemos JA, et al. 2013 ACCF/AHA guideline for the management of ST-elevation myocardial infarction: a report of the American College of Cardiology Foundation/American Heart Association Task Force on Practice Guidelines. Circulation. 2013;127(4):e362-425.

3. Lee WC, Fang HY, Chen $\mathrm{HC}$, Chen $\mathrm{CJ}$, Yang $\mathrm{CH}$, Hang $\mathrm{CL}$, et al. Anemia: A significant cardiovascular mortality risk after ST-segment elevation myocardial infarction complicated by the comorbidities of hypertension and kidney disease. PLOS ONE. 2017;12(7):e0180165.

4. Hong D, Choi KH, Song YB, Lee JM, Park TK, Yang JH, et al. Prognostic implications of post-percutaneous coronary intervention neutrophil-tolymphocyte ratio on infarct size and clinical outcomes in patients with acute myocardial infarction. Sci Rep. 2019;9(1):9646.

5. Moon J, Kang W, Oh PC, Seo SY, Lee K, Han SH, et al. Serum transaminase determined in the emergency room predicts outcomes in patients with acute ST-segment elevation myocardial infarction who undergo primary percutaneous coronary intervention. Int J Cardiol. 2014;177(2):442-7.

6. Jang HJ, Oh PC, Moon J, Suh J, Park HW, Park SD, et al. Prognostic impact of combined dysglycemia and hypoxic liver injury on admission in patients with ST-segment elevation myocardial infarction who underwent primary percutaneous coronary intervention (from the INTERSTELLAR Cohort). Am J Cardiol. 2017;119(8):1179-85.

7. Duffy BK, Gurm HS, Rajagopal V, Gupta R, Ellis SG, Bhatt DL. Usefulness of an elevated neutrophil to lymphocyte ratio in predicting long-term mortality after percutaneous coronary intervention. Am J Cardiol. 2006;97(7):993-6.

8. Uthamalingam S, Patvardhan EA, Subramanian S, Ahmed W, Martin W, Daley $\mathrm{M}$, et al. Utility of the neutrophil to lymphocyte ratio in predicting long-term outcomes in acute decompensated heart failure. Am J Cardiol. 2011;107(3):433-8.

9. Kurek T, Lenarczyk R, Kowalczyk J, Swiatkowski A, Kowalski O, StabrylaDeska J, et al. Effect of anemia in high-risk groups of patients with acute myocardial infarction treated with percutaneous coronary intervention. Am J Cardiol. 2010;105(5):611-8.

10. Moon J, Suh J, Oh PC, Lee K, Park HW, Jang HJ, et al. Relation of stature to outcomes in korean patients undergoing primary percutaneous coronary intervention for acute ST-Elevation myocardial infarction (from the INTERSTELLAR Registry). Am J Cardiol. 2016;118(2):177-82.

11. Oh PC, Lee K, Kim TH, Moon J, Park HW, Jang HJ, et al. Prognostic impact of alkaline phosphatase measured at time of presentation in patients undergoing primary percutaneous coronary intervention for ST-segment elevation myocardial infarction. PLoS ONE. 2017;12(2):e0171914.

12. Oh PC, Eom YS, Moon J, Jang HJ, Kim TH, Suh J, et al. Prognostic impact of the combination of serum transaminase and alkaline phosphatase determined in the emergency room in patients with ST-segment elevation myocardial infarction undergoing primary percutaneous coronary intervention. PLoS ONE. 2020;15(5):e0233286.

13. Barbero U, D'Ascenzo F, Nijhoff F, Moretti C, Biondi-Zoccai G, Mennuni $M$, et al. Assessing risk in patients with stable coronary disease: when should we intensify care and follow-up? Results from a meta-analysis of observational studies of the COURAGE and FAME Era. Scientifica (Cairo). 2016:2016:3769152

14. Stranders I, Diamant M, van Gelder RE, Spruijt HJ, Twisk JW, Heine RJ, et al. Admission blood glucose level as risk indicator of death after myocardial infarction in patients with and without diabetes mellitus. Arch Intern Med. 2004;164(9):982-8.

15. Yang SW, Zhou YJ, Hu DY, Nie XM, Liu YY, Hua Q, et al. Association between admission hypoglycaemia and in-hospital and 3-year mortality in older patients with acute myocardial infarction. Heart. 2010;96(18):1444-50.

16. van Toorenburg M, van den Berg VJ, van der Ploeg T, Heestermans AA, Dirksen MT, Hautvast RW, et al. Addition of routinely measured blood biomarkers significantly improves GRACE risk stratification in patients with myocardial infarction. Int J Cardiol. 2018;273:237-42.
17. Yanishi K, Nakamura T, Nakanishi N, Yokota I, Zen K, Yamano T, et al. A simple risk stratification model for ST-elevation myocardial infarction (STEMI) from the combination of blood examination variables: acute myocardial infarction-Kyoto Multi-Center Risk Study Group. PLoS ONE. 2016;11(11):e0166391.

18. Lofthus DM, Stevens SR, Armstrong PW, Granger CB, Mahaffey KW. Pattern of liver enzyme elevations in acute ST-elevation myocardial infarction. Coron Artery Dis. 2012;23(1):22-30.

19. Reinstadler SJ, Reindl M, Feistritzer HJ, Klug G, Mayr A, Kofler M, et al. Prognostic significance of transaminases after acute ST-elevation myocardial infarction: insights from a cardiac magnetic resonance study. Wien Klin Wochenschr. 2015;127(21-22):843-50.

20. Kosiborod M, Rathore SS, Inzucchi SE, Masoudi FA, Wang Y, Havranek EP, et al. Admission glucose and mortality in elderly patients hospitalized with acute myocardial infarction: implications for patients with and without recognized diabetes. Circulation. 2005;111(23):3078-86.

21. Williams SB, Goldfine AB, Timimi FK, Ting HH, Roddy MA, Simonson DC, et al. Acute hyperglycemia attenuates endothelium-dependent vasodilation in humans in vivo. Circulation. 1998;97(17):1695-701.

22. Stegenga ME, van der Crabben SN, Levi M, de Vos AF, Tanck MW, Sauerwein HP, et al. Hyperglycemia stimulates coagulation, whereas hyperinsulinemia impairs fibrinolysis in healthy humans. Diabetes. 2006;55(6):1807-12.

23. Esposito K, Nappo F, Marfella R, Giugliano G, Giugliano F, Ciotola M, et al. Inflammatory cytokine concentrations are acutely increased by hyperglycemia in humans: role of oxidative stress. Circulation. 2002;106(16):2067-72.

24. Chow E, Bernjak A, Williams S, Fawdry RA, Hibbert S, Freeman J, et al. Risk of cardiac arrhythmias during hypoglycemia in patients with type 2 diabetes and cardiovascular risk. Diabetes. 2014;63(5):1738-47.

25. Levy PS, Quigley RL, Gould SA. Acute dilutional anemia and critical left anterior descending coronary artery stenosis impairs end organ oxygen delivery. J Trauma. 1996;41(3):416-23.

26. Most AS, Ruocco NA Jr, Gewirtz H. Effect of a reduction in blood viscosity on maximal myocardial oxygen delivery distal to a moderate coronary stenosis. Circulation. 1986;74(5):1085-92.

27. Riley RF, Newby LK, Don CW, Alexander KP, Peterson ED, Peng SA, et al. Guidelines-based treatment of anaemic STEMI patients: practice patterns and effects on in-hospital mortality: a retrospective analysis from the NCDR. Eur Heart J Acute Cardiovasc Care. 2013;2(1):35-43.

28. Shacham Y, Gal-Oz A, Leshem-Rubinow E, Arbel Y, Flint N, Keren G, et al. Association of admission hemoglobin levels and acute kidney injury among myocardial infarction patients treated with primary percutaneous intervention. Can J Cardiol. 2015;31(1):50-5.

29. Halkin A, Singh M, Nikolsky E, Grines CL, Tcheng JE, Garcia E, et al. Prediction of mortality after primary percutaneous coronary intervention for acute myocardial infarction: the CADILLAC risk score. J Am Coll Cardiol. 2005;45(9):1397-405.

30. Park JS, Seo KW, Choi BJ, Choi SY, Yoon MH, Hwang GS, et al. Importance of prognostic value of neutrophil to lymphocyte ratio in patients with STelevation myocardial infarction. Medicine (Baltimore). 2018;97(48):e13471.

31. Han YC, Yang TH, Kim DI, Jin HY, Chung SR, Seo JS, et al. Neutrophil to lymphocyte ratio predicts long-term clinical outcomes in patients with ST-segment elevation myocardial infarction undergoing primary percutaneous coronary intervention. Korean Circ J. 2013:43(2):93-9.

32. Seropian IM, Toldo S, Van Tassell BW, Abbate A. Anti-inflammatory strategies for ventricular remodeling following ST-segment elevation acute myocardial infarction. J Am Coll Cardiol. 2014;63(16):1593-603.

33. Borekci A, Gur M, Turkoglu C, Baykan AO, Seker T, Sahin DY, et al. Neutrophil to lymphocyte ratio predicts left ventricular remodeling in patients with ST elevation myocardial infarction after primary percutaneous coronary intervention. Korean Circ J. 2016:46(1):15-22.

\section{Publisher's Note}

Springer Nature remains neutral with regard to jurisdictional claims in published maps and institutional affiliations. 